\title{
COMPACITY IN NARROW LIMIT TOWER SPACES
}

\author{
LIVIU C. FLORESCU
}

(Received January 2000 and in revised form 4 May 2000)

\begin{abstract}
We introduce a limit tower structure on the space of all bounded Radon measures on a completely regular space and we extend the Prohorov's theorem of narrow compactness. In the particular case of Polish spaces, we give a sequential version of this extension.
\end{abstract}

2000 Mathematics Subject Classification. 28A33, 46E27, 54E70, 54A20, 60B10.

1. Introduction. Let $T$ be a completely regular space, $\mathscr{B}$ the boreliens of $T$, and $2 \mathfrak{V}^{b}(T)$ the set of all bounded Radon measures on $(T, \mathscr{B})$ (i.e., the real bounded measures $\mu: \mathscr{B} \rightarrow \mathbb{R}$ such that $|\mu|(A)=\sup \{|\mu|(K): K$ is compact, $K \subseteq A\}$, for all $A \in \mathscr{B}$, where $|\mu|$ is the variation of $\mu$ ). Denote by $\mathscr{C}^{b}(T)$ the space of all bounded continuous real functions on $T$ and let $\|f\|=\sup \{|f(t)|: t \in T\}$, for every $f \in \mathscr{C}^{b}(T)$. We recall that a filter $\mathfrak{f}$ on $2 \mathfrak{D}^{b}(T)$ is narrowly convergent to $\mu$ if and only if $V_{\varepsilon, f}(\mu) \in \mathfrak{f}$, for all $f \in \mathscr{C}^{b}(T), \varepsilon>0$, where $V_{\varepsilon, f}(\mu)=\{v:|\mu(f)-v(f)|<\varepsilon\}$.

We say that a set $H \subseteq 2 \mathfrak{D}^{b}(T)$ is relatively narrowly compact if, for every filterbase $\mathfrak{2} \subseteq 2^{H}$ there exist a filter $\mathfrak{f}$ on $2 \mathfrak{W}^{b}(T)$ and $\mu \in \mathfrak{V}^{b}(T)$ such that $\mathfrak{f}$ converges to $\mu$.

Prohorov's classical theorem states that a bounded set $H \subseteq 2 \mathfrak{b}^{b}(T)$ is relatively narrowly compact if the following condition is satisfied:

$$
\forall \varepsilon>0, \quad \exists K_{\varepsilon} \text { compact } \subseteq T:|\mu|\left(T \backslash K_{\varepsilon}\right)<\varepsilon, \forall \mu \in H
$$

A set $H$ as in (1.1) is called tight.

We remark that, if $T$ is a Polish space (i.e., $T$ is a separable, completely metrizable space) or $T$ is a locally compact space, the converse is also true (relative narrow compactness implies tightness) (see [2, Section 5, Theorems 1 and 2]).

Limit tower spaces were first defined in 1997 by Kent and Brock [3] as an isomorphic gradated variant of convergence approach spaces of Löwen [8].

In this paper, we introduce on $2 \mathfrak{b}^{b}(T)$ a limit tower structure $\bar{p}=\left\{p_{a}: a \in[0,+\infty]\right\}$ (see [3]), where $p_{0}$ is the narrow convergence structure. Then, for every bounded set $H \subseteq \mathfrak{W}^{b}(T)$, there exists a number $t=t(H) \geq 0$ such that, for every filterbase $\mathfrak{2} \subseteq 2^{H}$ there exists a filter $\mathfrak{f}$ on $\mathfrak{2 N}^{b}(T), \mathfrak{2} \subseteq \mathfrak{f}, p_{t}$-convergent in $\mathfrak{2}^{b}(T)$ (see Theorem 3.8); we say that $H$ is $p_{t}$-relatively compact. The number $t(H)$ estimates the degree of tightness of $H$. If $H$ is tight then $t(H)=0$, so we obtain Prohorov's theorem.

If $T$ is a locally compact space we extend also the converse of Prohorov's theorem (see Theorem 3.12).

We give some examples in the particular case of $T=\mathbb{N}$ when $2 \mathfrak{W}^{b}(\mathbb{N})=\ell^{1}$. 
In Section 4, we obtain a sequential version of Theorem 3.8 on the subset $2 \mathrm{D}^{1}(T) \subseteq$ $2 \mathfrak{W}^{b}(T)$ of all probabilities on the Polish space $T$. So, every sequence $\left(\mu_{n}\right)_{n \in \mathbb{N}} \subseteq 2 \mathfrak{W}^{1}(T)$

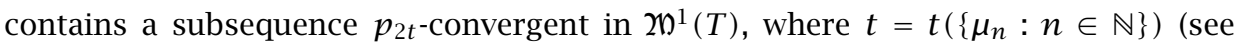
Theorem 4.9). In particular, we prove that the limit tower structure $\bar{p}$ on the set of probabilities is induced by a probabilistic metric on this space.

2. Limit tower structures. Let $X$ be a set, $\mathbb{B}(X)$ the set of all filterbases on $X$, and $2^{X}$ the power set of $X$; for every $\mathfrak{f} \in \mathbb{B}(X), \mathfrak{f}^{\prime}$ is the filter generated by $\mathfrak{f}$. For $x \in X$, let $\dot{x}$ denote the fixed ultrafilter generated by $\{x\}$.

DEFINITION 2.1 (see [3, Definition 1]). A limit structure on $X$ is a function $q: \mathbb{B}(X) \rightarrow$ $2^{X}$ satisfying

$$
\begin{gathered}
x \in q(\dot{x}), \quad x \in X, \\
q(\mathfrak{f})=q\left(\mathfrak{f}^{\prime}\right), \quad \forall \mathfrak{f} \in \mathbb{B}(X), \\
q\left(\mathfrak{f}^{\prime} \cap \mathfrak{G}^{\prime}\right)=q(\mathfrak{f}) \cap q(\mathfrak{G}), \quad \forall \mathfrak{f}, \mathfrak{G} \in \mathbb{B}(X) .
\end{gathered}
$$

A pair $(X, q)$, where $q$ is a limit structure on $X$ is called a limit space.

REMARK 2.2. The statement " $x \in q(\mathfrak{f})$ " will be written $\mathfrak{f} \stackrel{q}{\rightarrow} x$ and we say that $\mathfrak{f}$ $q$-converges to $x$.

REMARK 2.3. In [3], a limit structure is a function $q: \mathbb{F}(X) \rightarrow 2^{X}$, where $\mathbb{F}(X)$ denotes the set of all filters on $X$, satisfying

$$
\begin{aligned}
& x \in q(\dot{x}), \quad x \in X, \\
& \mathfrak{f} \subseteq \mathfrak{G} \Longrightarrow q(\mathfrak{f}) \subseteq q(\mathfrak{G}), \\
& x \in q(\mathfrak{f}) \Longrightarrow x \in q(\mathfrak{f} \cap \dot{x}), \\
& x \in q(\mathfrak{f}) \cap q(\mathfrak{G}) \Longrightarrow x \in q(\mathfrak{f} \cap \mathfrak{G}) .
\end{aligned}
$$

If we extend such a function $q$ to $\mathbb{B}(X)$ letting $q(\mathfrak{f})=q\left(\mathfrak{f}^{\prime}\right)$, then (2.4) is equivalent to (2.1), (2.2), and (2.3).

REMARK 2.4. If $\tau$ is a topology on $X$ and we define $\mathfrak{f} \stackrel{q_{\tau}}{\longrightarrow} x$ if and only if $\mathscr{V}_{\tau}(x) \subseteq \mathfrak{f}^{\prime}$, then $q_{\tau}$ is a limit structure on $X$ (here $\mathscr{V}_{\tau}(x)$ denotes the neighborhood filter of $x$ in $(X, \tau))$. More exactly we have the following proposition.

Proposition 2.5 (see [3, Proposition 2]). Let q be a limit structure on $X$; the necessary and sufficient condition for a topology $\tau$ to exist on $X$, such that $q=q_{\tau}$, is that $q$ fulfills the following condition:

(F) Let $\left\{\mathfrak{f}_{j}: j \in J\right\}$ be a family of filterbases on $X$ and $\left\{x_{j}: j \in J\right\} \subset X$ be such that $\mathfrak{f}_{j} \stackrel{q}{\rightarrow} x_{j}$ for all $j \in J$.

If $\Phi \in \mathbb{B}(J)$ is such that $\mathfrak{f} \stackrel{q}{\rightarrow} x$, where $\mathfrak{f}=\left\{\left\{x_{j}: j \in \phi\right\}: \phi \in \Phi\right\}$, then

$$
\bigcup_{\phi \in \Phi} \bigcap_{j \in \phi} \mathfrak{f}_{j}^{\prime} \stackrel{q}{\longrightarrow} x
$$


Definition 2.6 (see [3, Definition 4]). A limit tower $\bar{p}$ on a set $X$ is a family $\bar{p}=$ $\left\{p_{a}: a \in[0,+\infty]\right\}$ of limit structures on $X$ satisfying the following conditions:

$$
\begin{aligned}
& p_{a}(\mathfrak{f}) \subseteq p_{b}(\mathfrak{f}), \quad \forall a \leq b, \forall \mathfrak{f} \in \mathbb{B}(X), \\
& p_{\infty}(\mathfrak{f})=X, \quad \forall \mathfrak{f} \in \mathbb{B}(X), \\
& p_{a}(\mathfrak{f})=\cap_{b>a} p_{b}(\mathfrak{f}), \quad \forall a \in[0,+\infty), \forall \mathfrak{f} \in \mathbb{B}(X) .
\end{aligned}
$$

If $x \in p_{a}(\mathfrak{f})$, we say that $\mathfrak{f}$ is $p_{a}$-convergent to $x$ and we denote this by $\mathfrak{f} \stackrel{p_{a}}{\longrightarrow} x$. If $\bar{p}$ is a limit tower on $X,(X, \bar{p})$ is called a limit tower space.

The axiom (F) defined in Proposition 2.5 has a natural extension to a limit tower space $(X, \bar{p})$ :

(F) Let $a, b \in[0,+\infty],\left\{\mathfrak{f}_{j}: j \in J\right\} \subseteq \mathbb{B}(X)$, and $\left\{x_{j}: j \in J\right\} \subseteq X$ such that $\mathfrak{f}_{j} \stackrel{p_{a}}{\longrightarrow} x_{j}$, for all $j \in J$. If $\Phi \in \mathbb{B}(J)$ is such that $\mathfrak{f} \stackrel{p_{b}}{\longrightarrow} x$, where $\mathfrak{f}=\left\{\left\{x_{j}: j \in \phi\right\}: \phi \in \Phi\right\}$, then

$$
\bigcup_{\phi \in \Phi} \bigcap_{j \in \phi} \mathfrak{f}_{j}^{\prime} \stackrel{p_{a+b}}{\longrightarrow} x \text {. }
$$

DefinITION 2.7. A limit tower $\bar{p}$ on $X$ which satisfies (F) is called a topological limit tower.

REMARK 2.8. From [3, Theorems 9, 13 and Proposition 12(b)] we know that a topological limit tower is an isomorphic form of a Löwen's approach structure (see [8]).

3. Narrow limit tower on $2 \mathfrak{b}^{b}(T)$. In this section, we introduce a topological limit tower $\bar{p}=\left\{p_{a}: a \in[0,+\infty]\right\}$ on the space of bounded Radon measures on a completely regular space such that $p_{0}$-convergence is just the narrow convergence; then we extend the Prohorov's theorem of narrow compactness.

Let $T$ be a completely regular space, let $\mathscr{R}$ be the $\sigma$-algebra of Borel subsets of $T$, and let $2 \mathfrak{W}^{b}(T)$ be the set of all bounded Radon measures on $(T, \mathscr{B})$. Denote by $C^{b}(T)$ the set of all bounded continuous real functions on $T$. For every $f \in C^{b}(T)$ and $\mu \in \mathfrak{2}^{b}(T)$, we denote $\mu(f)=\int_{T} f d \mu$.

Now, for every $a \in[0,+\infty], \mu \in \mathfrak{2}^{b}(T)$, and $f \in C^{b}(T)$, we denote

$$
V_{a, f}(\mu)=\left\{\nu \in \mathfrak{2}^{b}(T):|\mu(f)-v(f)| \leq a\|f\|\right\} .
$$

Then, for every $a \in[0,+\infty)$, let $p_{a}: \mathbb{B}\left(\mathfrak{2}^{b}(T)\right) \rightarrow 2^{2 \mathfrak{M}^{b}(T)}$ defined by

$$
p_{a}(\mathfrak{f})=\left\{\mu \in \mathfrak{V}^{b}(T): \forall b>a, \forall \in C^{b}(T), V_{b, f}(\mu) \in \mathfrak{f}^{\prime}\right\},
$$

for all filterbases $\mathfrak{f}$ on $2 \mathfrak{W}^{b}(T)$; let $p_{\infty}$ be the indiscrete convergence structure on $2 \mathfrak{W}^{b}(T)$ $\left(p_{\infty}(\mathfrak{f})=2 \mathfrak{W}^{b}(T)\right.$, for all $\left.\mathfrak{f} \in \mathbb{B}\left(2 \mathfrak{W}^{b}(T)\right)\right)$.

We remark that $\mathfrak{f} \stackrel{p_{a}}{\longrightarrow} \mu$ if and only if for all $b>a$, for all $f \in C^{b}(T), V_{b, f}(\mu) \in \mathfrak{f}^{\prime}$.

Proposition 3.1. The limit tower $\bar{p}=\left\{p_{a}: a \in[0,+\infty]\right\}$ is a topological limit tower on $2 \mathrm{~V}^{b}(T)$.

Proof. For every $a \in[0,+\infty), \mu \in \mathfrak{2}^{b}(T)$, and $f \in C^{b}(T)$, we have $\mu \in V_{a, f}(\mu)$, so that we have (2.1). 
Equations (2.2), (2.3), (2.6), and (2.7) are consequences of the definition of $\bar{p}$. From (2.6), $p_{a}(\mathfrak{f}) \subseteq \bigcap_{b>a} p_{b}(\mathfrak{f})$, for all $\mathfrak{f} \in \mathbb{B}\left(2 \mathfrak{W}^{b}(T)\right)$. If $\mathfrak{f} \stackrel{p_{c}}{\longrightarrow} \mu$, for all $c>a$, then for all $b>a$, there exists $c$ such that $a<c<b$ hence $V_{b, f}(\mu) \in \mathfrak{f}$, for all $f \in C^{b}(T)$. Therefore $\mathfrak{f} \stackrel{p_{a}}{\longrightarrow} \mu$ and so we have (2.8).

(F) Let $a, b \geq 0,\left\{\mathfrak{f}_{j}: j \in J\right\} \subseteq \mathbb{B}\left(\mathfrak{W W}^{b}(T)\right)$, and $\left\{\mu_{j}: j \in J\right\} \subseteq \mathfrak{W}^{b}(T)$ such that (1) $\mathfrak{f}_{j} \stackrel{p_{a}}{\longrightarrow} \mu_{j}$, for all $j \in J$. Let $\Phi$ be a filterbase on $J$ such that (2) $\mathfrak{f} \stackrel{b}{\longrightarrow} \mu$, where $\mathfrak{f}=$ $\left\{\left\{\mu_{j}\right\}_{j \in \phi}: \phi \in \Phi\right\}$. Then for all $u>a+b$, there exist $d>a, e>b$ such that $u=d+e$. Then for all $f \in C^{b}(T)$, from (2), $V_{e, f}(\mu) \in \mathfrak{f}^{\prime}$ hence, there exists $\phi \in \Phi$ such that $\left\{\mu_{j}\right\}_{j \in \phi} \subseteq V_{e, f}(\mu)$. Then (3) $\left|\mu_{j}(f)-\mu(f)\right| \leq e\|f\|$, for all $j \in \phi$.

From (1), for all $j \in J, V_{d, f}\left(\mu_{j}\right) \in \mathfrak{f}_{j}^{\prime}$. But, from (3), $V_{d, f}\left(\mu_{j}\right) \subseteq V_{u, f}(\mu)$, so that $V_{u, f} \in$ $\mathfrak{f}_{j}^{\prime}$, for all $j \in \phi$. Therefore,

$$
V_{u, f}(\mu) \in \bigcap_{j \in \phi} \mathfrak{f}_{j}^{\prime} \subseteq \bigcup_{\phi \in \Phi} \bigcap_{j \in \phi} \mathfrak{f}_{j}^{\prime} .
$$

It follows that $\mu \in p_{a+b}\left(\bigcup_{\phi \in \Phi} \bigcap_{j \in \phi} \mathfrak{f}_{j}^{\prime}\right)$, so that $\bar{p}=\left\{p_{a}: a \in[0,+\infty]\right\}$ is a topological limit tower on $2 \mathfrak{N}^{b}(T)$.

Definition 3.2. We say that $\bar{p}=\left\{p_{a}: a \in[0,+\infty]\right\}$ is the narrow limit tower on $2 \mathrm{~N}^{b}(T)$.

REMARK 3.3. Note that $p_{0}$ is the narrow convergence structure on $2 \mathfrak{W}^{b}(T)$. Indeed, $\mathfrak{f} \stackrel{p_{0}}{\longrightarrow} \mu$ if and only if for all $\varepsilon>0$, for all $f \in C^{b}(T), V_{\varepsilon, f} \in \mathfrak{f}^{\prime}$. But the sets $V_{\varepsilon, f}(\mu)=$ $\{v:|\mu(f)-v(f)| \leq \varepsilon\|f\|\}$ form a subbase for the neighbourhood system of $\mu$ in the narrow topology on $2 \mathfrak{b}^{b}(T)$; so that $\mathfrak{f}$ is narrowly convergent to $\mu$.

REMARK 3.4. If $\mathfrak{f} \stackrel{p_{a}}{\longrightarrow} \mu$ then $\mathfrak{f} \stackrel{p_{b}}{\longrightarrow} \mu$, for all $b \geq a$. Thus $p_{0}$ is the finest limit structure of $\bar{p}$.

REMARK 3.5. We may interpret $\inf \left\{a: f \stackrel{p_{a}}{\longrightarrow} \mu\right\}$ as the degree of narrow convergence of filterbase $\mathfrak{f}$ to $\mu$.

REMARK 3.6. For every net $\left(\mu_{i}\right)_{i \in I} \subseteq \mathfrak{2}^{b}(T)\left((I, \leq)\right.$ is a directed set) let $\mathfrak{f}=\left\{\left\{x_{j}:\right.\right.$ $j \geq i\}: i \in I\}$ be the filterbase generated by $\left(\mu_{i}\right)_{i \in I}$.

If $\bar{p}=\left\{p_{a}: a \in[0,+\infty]\right\}$ is the narrow limit tower on $2 \mathfrak{N}^{b}(T)$, then we say that $\mu_{i} \stackrel{a}{\longrightarrow} \mu$ if $\mathfrak{f} \stackrel{p_{a}}{\longrightarrow} \mu$. Therefore, $\mu_{i} \stackrel{a}{\longrightarrow} \mu$ if and only if

$$
\limsup _{i}\left|\mu_{i}(f)-\mu(f)\right| \leq a \cdot\|f\|, \quad \forall f \in C^{b}(T) .
$$

DEFINITION 3.7. We say that a subset $H \subseteq 2 \mathrm{D}^{b}(T)$ is a-relatively compact if for every filterbase $\mathfrak{B} \subseteq 2^{H}$, there exist a filter $\mathfrak{f}$ on $\mathfrak{2}^{b}(T)$ and $\mu \in \mathfrak{2}^{b}(T)$ such that $\mathfrak{B} \subseteq \mathfrak{f}$ and $\mathfrak{f} \stackrel{p_{a}}{\longrightarrow} \mu$.

We remark that $H$ is 0 -relatively compact if and only if $H$ is relatively narrowly compact.

A subset $H \subseteq 2 \mathfrak{D}^{b}(T)$ is bounded if $\sup \{|\mu|(T): \mu \in H\}<+\infty$, where $|\mu|$ is the variation of $\mu$. The mapping $\mu \mapsto|\mu|(T)=\|\mu\|$ is a norm on $2 \mathfrak{D}^{b}(T)$.

Let $\mathscr{K}(T)$ be the family of all compact sets on $T$; for every bounded set $H \subseteq 2 \mathfrak{V}^{b}(T)$ 
we denote

$$
t(H)=\inf _{K \in \mathscr{K}(T)} \sup _{\mu \in H}|\mu|(T \backslash K) .
$$

We remark that $t(H) \in[0,+\infty)$ and $t(H)=0$ if and only if $H$ is tight. We say that $t(H)$ is the degree of tightness of $H$.

Now we give an extension of Prohorov's theorem.

THEOREM 3.8. Every bounded set $H \subseteq 2 \mathfrak{W}^{b}(T)$ is $t(H)$-relatively compact.

Proof. Let $X$ be the Stone-Čech compactification of $T$ and $i: T \rightarrow X$ be the canonical injection of $T$ in $X$. We remark that $C^{b}(X)=C(X)\left(X\right.$ is compact); so $\left(2 \mathrm{~V}^{b}(X),\|\cdot\|\right)$ is the topological dual of the Banach space $(C(X),\|\cdot\|)$ and the narrow topology on $2 \mathfrak{N}^{b}(X)$ is the weak*-topology, $w^{*}$, of this dual space.

For every $\mu \in \mathfrak{2}^{b}(T)$ we define $v=I(\mu) \in \mathfrak{2 D}^{b}(X)$, where $I(v)(F)=\mu(F \circ i)$, for every $F \in C(X) ;\|v\|=|v|(X)=|\mu|(T)=\|\mu\|$ so that $I: 2 \hat{W}^{b}(T) \rightarrow 2 \mathfrak{W}^{b}(X), \mu \mapsto I(\mu)$, is an isometric embedding.

Let $H$ be a bounded subset of $2 \mathfrak{b}^{b}(T)$; then $I(H)$ is a bounded subset of $2 \mathfrak{b}^{b}(X)$. Therefore $I(H)$ is $w^{*}$-relatively compact. For every filterbase $\mathfrak{B} \subseteq 2^{H}, I(\mathfrak{B})=\{I(B)$ : $B \in \mathfrak{b}\}$ is a filterbase on $I(H)$. So that, there exists a filter $\mathfrak{G}$ on $\mathfrak{2 b}^{b}(X) w^{*}$-convergent to a measure $v_{0} \in \mathfrak{2 \mathfrak { b }}^{b}(X)$ such that $I(\mathfrak{B}) \subseteq \mathfrak{G}$. From the definition of $t(H)$, there exists a sequence $\left(K_{n}\right)_{n} \subseteq \mathscr{K}(T)$ such that (1) $|\mu|\left(T \backslash K_{n}\right)<t(H)+1 / n$, for all $n \in \mathbb{N}$, for all $\mu \in H$. We denote $T_{0}=\bigcup_{n=1}^{\infty} K_{n}$ and (2) $X_{0}=\bigcup_{n=1}^{\infty} i\left(K_{n}\right)=i\left(T_{0}\right)$.

For every $n \in \mathbb{N}, i\left(K_{n}\right) \in \mathscr{K}(X)$, so that $X_{0}$ is a Borel set of $X$. On the other hand, for every $n \in \mathbb{N}, X \backslash i\left(K_{n}\right)$ is an open subset of $X$ so that the mapping $\lambda \mapsto|\lambda|\left(X \backslash i\left(K_{n}\right)\right)$ is a $w^{*}$-lower semi-continuous mapping on $2 \mathfrak{W}^{b}(X)$ (see [2, Section 5, Proposition 6(a)]).

From $\mathfrak{G} \stackrel{w^{*}}{\longrightarrow} v_{0}$, for all $n \in \mathbb{N}$, there exists $G_{n} \in \mathfrak{G}$ such that (3) $\left|\nu_{0}\right|\left(X \backslash i\left(K_{n}\right)\right)-1 / n<$ $|\lambda|\left(X \backslash i\left(K_{n}\right)\right)$, for all $\lambda \in G_{n}$.

The filterbase $\mathfrak{2}$ is a filterbase on $H$ so that $\mathfrak{2} \neq \varnothing$. Let $B_{0}$ be a set in $\mathfrak{2}$; then $I\left(B_{0}\right) \in$ $I(23) \subseteq \mathfrak{6}$.

For every $n \in \mathbb{N}$, there exists $\mu_{n} \in B_{0}$ such that $I\left(\mu_{n}\right) \in G_{n}\left(I\left(B_{0}\right) \cap G_{n} \neq \varnothing\right)$. Therefore, from (1) and (3), for every $n \in \mathbb{N}$,

$$
\begin{aligned}
\left|v_{0}\right|\left(X \backslash X_{0}\right) & \leq\left|v_{0}\right|\left(X \backslash i\left(K_{n}\right)\right)<\left|I\left(\mu_{n}\right)\right|\left(X \backslash i\left(K_{n}\right)\right)+\frac{1}{n} \\
& =\left|\mu_{n}\right|\left(i^{-1}\left(X \backslash i\left(K_{n}\right)\right)\right)+\frac{1}{n}=\left|\mu_{n}\right|\left(T \backslash K_{n}\right)+\frac{1}{n}<t(H)+\frac{2}{n} .
\end{aligned}
$$

Hence (4) $\left|v_{0}\right|\left(X \backslash X_{0}\right) \leq t(H)$.

Now, $X$ being the Stone-Čech compactification of $T$, for every $f \in C^{b}(T)$ there exists $F \in C(X)$ such that $F \circ i=f$ and $\|F\|=\|f\|$ (see [10, Theorem 1.4.6, page 25]). Now we define $J: C^{b}(T) \rightarrow \mathbb{R}$ letting

$$
J(f)=v_{0}\left(F \cdot \chi_{X_{0}}\right)=\int_{X_{0}} F d v_{0} .
$$

Obviously, $J$ is a continuous linear mapping on $C^{b}(T)$. For every $\varepsilon>0$, from (2), there exists $K \in \mathscr{K}(T)$ such that $\left|\nu_{0}\right|\left(X_{0} \backslash i(K)\right)<\varepsilon$ and $i(K) \subseteq X_{0}$. Then, for every $g \in C^{b}(T)$ 
with $|g| \leq 1$ and $\left.g\right|_{K}=0$, let $G \in C(X)$ such that $G \circ i=g$. Therefore, we have

$$
\begin{aligned}
|J(g)|=\left|v_{0}\left(G \cdot X_{X_{0}}\right)\right| & \leq\left|v_{0}\left(G \cdot X_{X_{0} \backslash i(K)}\right)\right|+\left|v_{0}\left(G \cdot X_{i(K)}\right)\right| \\
& \leq\left|v_{0}\right|\left(X_{0} \backslash i(K)\right)<\varepsilon .
\end{aligned}
$$

Hence, $J$ is a linear mapping satisfying the condition $(M)$ from [2, Section 5 , Proposition 5] so that there exists exactly one measure $\mu_{0} \in 2 \mathfrak{W}^{b}(T)$ such that $\mu_{0}(f)=J(f)$, for every $f \in C^{b}(T)$. Then we have (5) $\mu_{0}(f)=v_{0}\left(F \cdot \chi_{X_{0}}\right)$, for all $f \in C^{b}(T)$, where $F$ is the continuous extension of $f$ to $X$.

Now, for every $f_{1}, \ldots, f_{n} \in C^{b}(T)$ with $\left\|f_{k}\right\|>0$, for all $k=1, \ldots, n$, let $F_{1}, \ldots, F_{n} \in$ $C(X)$ such that $F_{k} \circ i=f_{k}$ and $\left\|F_{k}\right\|=\left\|f_{k}\right\|$, for every $k=1, \ldots, n$.

For all $b>t(H)$, let $\varepsilon=(b-t(H)) \cdot \min \left\{\left\|f_{k}\right\|: k=1, \ldots, n\right\}>0$. The set

$$
G=\bigcap_{k=1}^{n}\left\{\lambda \in \mathfrak{2}^{b}(X):\left|\lambda\left(F_{k}\right)-v_{0}\left(F_{k}\right)\right|<\varepsilon\right\}
$$

is a $w^{*}$-neighborhood of $v_{0}$ and so is a member of $\mathfrak{E}\left(\mathfrak{G} \stackrel{w^{*}}{\longrightarrow} v_{0}\right)$. Therefore, for every $B \in \mathfrak{B}, G \cap I(B) \neq \varnothing(I(\mathfrak{B}) \subseteq \mathfrak{G})$. Hence there exists $\mu \in B$ such that $I(\mu) \in G$. Then, for every $k=1, \ldots, n$, from (4) and (5), we have

$$
\begin{aligned}
\left|\mu\left(f_{k}\right)-\mu_{0}\left(f_{k}\right)\right| & =\left|\mu\left(F_{k} \circ i\right)-\mu_{0}\left(f_{k}\right)\right|=\left|I(\mu)\left(F_{k}\right)-v_{0}\left(F_{k} \cdot x_{X_{0}}\right)\right| \\
& \leq\left|I(\mu)\left(F_{k}\right)-v_{0}\left(F_{k}\right)\right|+\left|v_{0}\left(F_{k} \cdot x_{X \backslash X_{0}}\right)\right|<\varepsilon+\| F_{k}|| \cdot\left|v_{0}\right|\left(X \backslash X_{0}\right) \\
& <\varepsilon+\left\|f_{k}|| \cdot t(H) \leq(b-t(H)) \cdot\right\| f_{k}\|+\| f_{k}\|\cdot t(H)=b \cdot\| f_{k} \| .
\end{aligned}
$$

Therefore, $\mu \in \bigcap_{k=1}^{n} V_{b, f_{k}}\left(\mu_{0}\right)$. So, for every $b>t(H), n \in \mathbb{N}, f_{1}, \ldots, f_{n} \in C^{b}(T)$ and $B \in \mathfrak{B}$,

$$
\bigcap_{k=1}^{n} V_{b, f_{k}}\left(\mu_{0}\right) \cap B \neq \varnothing .
$$

Let $\mathfrak{f}$ be the filter generated by the filterbase

$$
\left\{\bigcap_{k=1}^{n} V_{b, f_{k}}\left(\mu_{0}\right) \cap B: b>t(H), f_{1}, \ldots, f_{n} \in C^{b}(T), B \in \mathfrak{2}\right\} .
$$

Then $\mathfrak{2} \subseteq \mathfrak{f}$ and $\mathfrak{f} \stackrel{p_{t(H)}}{\longrightarrow} \mu_{0}$, so that $H$ is a $t(H)$-relatively compact set.

REMARK 3.9. If $H$ is tight in $2 \boldsymbol{b}^{b}(T)$ then $t(H)=0$, so that $H$ is a relatively narrowly compact set and we obtain Prohorov's theorem.

REMARK 3.10. Let $a \geq b \geq 0$; then, every $b$-relatively compact set is $a$-relatively compact set, also. Therefore, for every bounded set $H \subseteq 2 \mathfrak{D}^{b}(T)$

$$
[t(H),+\infty) \subseteq\{a \geq 0: H \text { is } a \text {-relatively compact }\} .
$$

REMARK 3.11. We say that $H \subseteq 2 \mathfrak{W}_{+}^{b}(T)$ is a-relatively compact in $2 \mathfrak{W}_{+}^{b}(T)$ if, for every filterbase $\mathfrak{2} \subseteq 2^{H}$, there exist a filter $\mathfrak{f}$ on $\mathfrak{W}_{+}^{b}(T)$ and $\mu \in \mathfrak{W}_{+}^{b}(T)$ such that $\mathfrak{B} \subseteq \mathfrak{f}$ and for all $b>a$, for all $f \in C^{b}(T), V_{b, f}(\mu) \cap 2 \mathfrak{W}_{+}^{b}(T) \in \mathfrak{f}$; we say in this case that $\mathfrak{f} \stackrel{p_{a}}{\longrightarrow} \mu$ in $2 \mathfrak{B D}_{+}^{b}(T)$. 
The subset of all positive measures, $2 \mathfrak{W}_{+}^{b}(X)$, is closed in the narrow topology of $2 \mathfrak{W}^{b}(X)$ (see [2, Section 5, Remark 2]) so that, if $H \subseteq \mathfrak{2}_{+}^{b}(T)$ is a bounded subset, then $I(H)$ is $w^{*}$-relatively compact in $2 W_{+}^{b}(X)$. Then we follow the proof of Theorem 3.8 and we obtain that every bounded subset $H \subseteq \mathfrak{W}_{+}^{b}(T)$ is $t(H)$-relatively compact in $2 \mathfrak{N}_{+}^{b}(T)$. Also, we have

$$
[t(H),+\infty) \subseteq\left\{a \geq 0: H \text { is } a \text {-relatively compact in } 2 \mathfrak{V}_{+}^{b}(T)\right\} .
$$

In the particular case where $T$ is locally compact, we have the converse of Theorem 3.8 in the subspace $2 \mathfrak{W}_{+}^{b}(T)$.

THEOREM 3.12. Let $T$ be a locally compact space and $H$ an a-relatively compact set in $2 \mathfrak{V}_{+}^{b}(T)$; then $t(H) \leq a$.

Proof. We suppose that $H$ is an $a$-relatively compact subset of $2 \mathrm{~V}_{+}^{b}(T)$ and $t(H)=$ $\inf _{K \in \mathscr{K}(T)} \sup _{\mu \in H} \mu(T \backslash K)>a$. Then, for every $\varepsilon>0$ and $K \in \mathscr{K}(T)$, there exists $\mu_{K} \in H$ such that (1) $\mu_{K}(T \backslash K)>a+\varepsilon$.

For every $K \in \mathscr{K}(T)$ we denote $B_{K}=\left\{\mu_{L}: L \in \mathscr{K}(T), K \subseteq L\right\}$. Then $\mathfrak{B}=\left\{B_{K}: K \in \mathscr{K}(T)\right\}$ is a filterbase on $H$ so that there exist a filter $\mathfrak{f}$ on $2 \mathfrak{W}_{+}^{b}(T)$ and $\mu \in \mathfrak{W}_{+}^{b}(T)$ such that $2 \mathfrak{b} \subseteq \mathfrak{f}$ and (2) $\mathfrak{f} \stackrel{p_{a}}{\longrightarrow} \mu$, in $\mathfrak{W}_{+}^{b}(T)$ (see Remark 3.11). Since $\mu$ is a Radon measure, there exists $K_{0} \in \mathscr{K}(T)$ such that (3) $\mu\left(T \backslash K_{0}\right)<\varepsilon / 2$.

Let $U$ be a relatively compact neighborhood of $K$ and $f: T \rightarrow[0,1]$ a continuous function such that (4) $\left.f\right|_{K_{0}}=0$ and $\left.f\right|_{T \backslash U}=1$.

We remark that $f \in C^{b}(T)$ and $\|f\|=1$. Now let $b=a+\varepsilon / 2>a$ and $f \in C^{b}(T)$; from (2), $V_{b, f}(\mu) \in \mathfrak{f} \supseteq \mathfrak{2}$ so that (5) $V_{b, f}(\mu) \cap B_{\bar{U}} \neq \varnothing$.

Hence, there exists $K \in \mathscr{K}(T), K \supseteq \bar{U}$ such that (6) $\left|\mu_{K}(f)-\mu(f)\right| \leq b \cdot\|f\|=b$.

From (1), (3), (4), and (6) we obtain the following contradiction:

$$
\begin{aligned}
a+\varepsilon<\mu_{K}(T \backslash K) & \leq \mu_{K}(T \backslash \bar{U}) \leq \mu_{K}(f) \leq \mu(f)+a+\frac{\varepsilon}{2} \\
& \leq \mu\left(T \backslash K_{0}\right)+a+\frac{\varepsilon}{2}<a+\varepsilon .
\end{aligned}
$$

REMARK 3.13. If $H$ is a relatively narrowly compact subset of $2 \hat{n}_{+}^{b}(T)$ (i.e., 0-relatively compact set), then $t(H)=0$ so that $H$ is tight. Therefore, we obtain the converse of Prohorov's theorem; so Theorem 3.12 is an extension of [2, Section 5, Theorem 2].

REMARK 3.14. From Remark 3.11 and Theorem 3.12, we obtain (in the case of locally compact spaces)

$$
[t(H),+\infty)=\left\{a \geq 0: H \text { is } a \text {-relatively compact in } 2 \mathfrak{N}_{+}^{b}(T)\right\} .
$$

EXAMPLE 3.15. Let $T=\mathbb{N}$ be the set of natural numbers and $2 \mathfrak{B}=\mathscr{P}(\mathbb{N})$. Then $2 \mathfrak{B}^{b}(\mathbb{N})=$ $\ell^{1}$ (the space of all sequences of real numbers $\left(x_{n}\right)_{n \in \mathbb{N}}$ such that $\sum_{n=1}^{\infty}\left|x_{n}\right|<+\infty$ ) and $C^{b}(T)=\ell^{\infty}$ (the space of all bounded sequences of real numbers). Indeed,

$$
\begin{aligned}
\forall x & =\left(x_{n}\right)_{n} \in \ell^{1}, x: \mathfrak{B} \longrightarrow \mathbb{R}, \quad x(A)=\sum_{n \in A} x_{n}, \\
x(y) & =\sum_{n} x_{n} y_{n}, \quad \forall y=\left(y_{n}\right)_{n} \in \ell^{\infty} .
\end{aligned}
$$


Let $\left(x^{p}\right)_{p \in \mathbb{N}} \subseteq 2 \mathfrak{D}^{b}(\mathbb{N})$ and $x \in \mathfrak{W}^{b}(\mathbb{N})$, where $x^{p}=\left(x_{n}^{p}\right)_{n}$, for every $p \in \mathbb{N}$ and $x=\left(x_{n}\right)_{n}$. Then $x^{p} \stackrel{a}{\longrightarrow} x$ if and only if (1) $\limsup _{p}\left|\sum_{n \in \mathbb{N}}\left(x_{n}^{p}-x_{n}\right) \cdot y_{n}\right| \leq a \cdot \sup _{n}\left|y_{n}\right|$, for all $\left(y_{n}\right)_{n} \in \ell^{\infty}$ (see Remark 3.6).

For every bounded set $H=\left\{x^{p}: p \in \mathbb{N}\right\} \subseteq \mathfrak{V}^{b}(\mathbb{N})(2) t(H)=\inf _{m} \sup _{p} \sum_{n=m}^{\infty}\left|x_{n}^{p}\right|$.

Let $\left(x_{p}\right)_{p \in \mathbb{N}} \subseteq[0,1]$ be a sequence; we define

$$
x_{n}^{p}= \begin{cases}1-x_{p}, & n=0, \\ x_{p}, & n=p, \\ 0, & \text { otherwise }\end{cases}
$$

Then $x^{p}=\left(x_{n}^{p}\right)_{n \in \mathbb{N}} \in \mathfrak{2 N}^{b}(\mathbb{N})$ and, from (2), we obtain

$$
t\left(\left\{x^{p}: p \in \mathbb{N}\right\}\right)=\limsup _{n} x_{n}=t .
$$

We easily remark that $x^{p} \stackrel{t}{\rightarrow} x$, where $x=\left(x_{n}\right)_{n}$ and

$$
x_{n}= \begin{cases}1, & n=0, \\ 0, & n>0 .\end{cases}
$$

From Remark 3.14, $\inf \left\{a \geq 0: x^{p} \stackrel{a}{\rightarrow} x\right\}=\limsup _{n} x_{n}$. If $x_{n} \rightarrow 0$, then $\left(x^{p}\right)_{p}$ is narrowly convergent to $x$. In the particular case where $x_{n}=1$, for every $n \in \mathbb{N}, x^{p}$ is the Dirac measure $\delta_{p}$ and $\delta_{p} \stackrel{1}{\rightarrow} \delta_{0}$.

We remark that

$$
\inf \left\{a \geq 0: \delta_{p} \stackrel{a}{\longrightarrow} \delta_{0}\right\}=1 .
$$

4. Probabilistic metric on $2 n^{1}(T)$. Let $(T, d)$ be a Polish space and let $2 n^{1}(T) \subseteq$ $2 \mathfrak{W}_{+}^{b}(T)$ be the subset of all probabilities on $T$. We say that a net $\left(\mu_{i}\right)_{i \in I} \subseteq 2 \mathfrak{W}^{1}(T)$ is $p_{a}$-convergent to $\mu \in \mathfrak{W W}^{1}(T)(a \geq 0)$ if

$$
\limsup _{i}\left|\mu_{i}(f)-\mu(f)\right| \leq a \cdot\|f\|, \quad \forall f \in C^{b}(T) .
$$

We denote this by $\mu_{i} \stackrel{a}{\rightarrow} \mu$. So, $\bar{p}=\left\{p_{a}: a \in[0,+\infty]\right\}$ is the narrow limit tower induced on $2 \mathfrak{W}^{1}(T)$ (see Remark 3.6). If $X$ is the Stone-Čech compactification of $T$, the subset $2 \mathfrak{W}^{1}(X)$ is a compact set of $2 \mathfrak{W}^{b}(X)$ (see [2, Section 5, Proposition 11]). So, with a similar argument to that of Remark 3.11, we deduce that every subset $H \subseteq 2 \mathfrak{W}^{1}(T)$ is $t(H)$ relatively compact in $2 \mathfrak{b}^{1}(T)$ (i.e., every net $\left(\mu_{i}\right)_{i \in I}$ has a subnet $p_{a}$-convergent).

Theorem 4.1 has a similar proof to that of Portmanteau's theorem (see [1, Theorem 2.1, Appendix III, Theorem 3]) which we omit.

THEOREM 4.1. Let $\left(\mu_{i}\right)_{i \in I}$ be a net in $2 \mathfrak{W}^{1}(T), \mu \in 2 \mathfrak{W}^{1}(T)$ and $a \geq 0$; the following statements are equivalent:

$$
\begin{gathered}
\mu_{i} \stackrel{a}{\longrightarrow} \mu, \\
\limsup _{i}\left|\mu_{i}(f)-\mu(f)\right| \leq a, \quad \forall f \in C^{b}(T) \text { with }\|f\| \leq 1,
\end{gathered}
$$




$$
\begin{gathered}
\limsup _{i} \mu_{i}(F) \leq \mu(F), \quad \forall F=\bar{F} \subseteq T, \\
\liminf _{i} \mu_{i}(D) \geq \mu(D), \quad \forall D=D^{\circ} \subseteq T, \\
\limsup _{i}\left|\mu_{i}(A)-\mu(A)\right| \leq a, \quad \forall A \in \mathscr{B} \text { with } \mu\left(\bar{A}-A^{\circ}\right)=0 .
\end{gathered}
$$

In Theorem 4.1, $\bar{A}$ and $A^{\circ}$ denote the closure and the interior of $A$ in the topological space $\left(T, \tau_{d}\right)$, respectively.

REMARK 4.2. In Theorem 4.1, we can suppose that $a \in[0,1]$.

REMARK 4.3. R. Löwen gave a similar result in [7, Theorem 6].

Definition 4.4. For every $F=\bar{F} \subseteq T$ and $\varepsilon>0$ we denote $F^{\varepsilon}=\{t \in T: d(t, F)<\varepsilon\}$. For every $a \in[0,1]$ we define $L_{a}: 2 \mathfrak{W}^{1}(T) \times 2 \mathfrak{W}^{1}(T) \rightarrow \mathbb{R}_{+}$letting

$$
L_{a}(\mu, v)=\inf \left\{\varepsilon>0: \mu(F)<v\left(F^{\varepsilon}\right)+a+\varepsilon, v(F)<\mu\left(F^{\varepsilon}\right)+a+\varepsilon, \forall F=\bar{F} \subseteq T\right\} .
$$

REMARK 4.5. $L_{0}$ is the metric of Lévy-Prohorov on $2 \mathrm{~W}^{1}(T)$. Therefore, $L_{0}$ induces the narrow topology on $2 W^{1}(T)$ and $\left(2 W^{1}(T), L_{0}\right)$ is a Polish space [2, Section 5, Examples 8 and 9].

REMARK 4.6. The family $\mathscr{L}=\left\{L_{a}: a \in[0,1]\right\}$ has the following properties:

$$
\begin{gathered}
L_{a}(\mu, v)=0, \quad \forall a \geq 0 \Longleftrightarrow \mu=v, \\
L_{a}(\mu, v)=L_{a}(\nu, \mu), \quad \forall \mu, v \in \mathfrak{V}^{1}(T), \quad \forall a \in[0,1], \\
L_{a+b}(\mu, \nu) \leq L_{a}(\mu, \lambda)+L_{b}(\lambda, v), \quad \forall \mu, \nu, \lambda \in \mathfrak{V}^{1}(T), \quad \forall a, b \in[0,1], \\
L_{a}(\mu, v)=\sup _{b>a} L_{b}(\mu, v), \quad \forall \mu, v \in \mathfrak{V}^{1}(T), \quad \forall a \in[0,1) .
\end{gathered}
$$

In [4, Theorem 1] we proved that such a family $\mathscr{L}$ is an equivalent gradated form of a probabilistic metric $\left(F, T_{m}\right)$, where, for every $\mu, v \in \mathfrak{V}^{1}(T)$ and $a>0$,

$$
F(\mu, v)(a)=\operatorname{supinf}_{\varepsilon>0}\left\{\min \left[\mu\left(F^{a-\varepsilon}\right)-v(F), \nu\left(F^{a-\varepsilon}\right)-\mu(F)\right]+1+a\right\} \wedge 1
$$

and $T_{m}(a, b)=\max \{a+b-1,0\}$. For the space of distribution functions, equivalent probabilistic metrics are introduced in $[5,6,9]$.

In Theorem 4.7 we compare the narrow limit tower with the convergence structures induced by the family of semi-pseudometrics $\mathscr{L}=\left\{L_{a}: a \in[0,1]\right\}$. So, this theorem is an important step to obtain a sequential version of Theorem 3.8.

THEOREM 4.7. Let $\left(\mu_{i}\right)_{i \in I}$ be a net in $2 \mathfrak{W}^{1}(T), \mu \in \mathfrak{W}^{1}(T)$ and $a \in[0,1]$.

$$
\begin{aligned}
& \text { If } L_{a}\left(\mu_{i}, \mu\right) \rightarrow 0, \quad \text { then } \mu_{i} \stackrel{a}{\rightarrow} \mu, \\
& \text { If } \mu_{i} \stackrel{a}{\rightarrow} \mu, \text { then } L_{2 a}\left(\mu_{i}, \mu\right) \longrightarrow 0 .
\end{aligned}
$$

Proof. (i) We suppose that $L_{a}\left(\mu_{i}, \mu\right) \rightarrow 0$; then, for every $n \in \mathbb{N}^{*}$, there exists $i_{n} \in I$ such that, for every $i \geq i_{n}, L_{a}\left(\mu_{i}, \mu\right)<1 / n$. Therefore,

$$
\mu_{i}(F)<\mu\left(F^{1 / n}\right)+a+\frac{1}{n}, \quad \forall F=\bar{F},
$$


so that, for every $F=\bar{F} \subseteq T$,

$$
\limsup _{i} \mu_{i}(F) \leq \sup _{i \geq i_{n}} \mu_{i}(F) \leq \mu\left(F^{1 / n}\right)+a+\frac{1}{n} .
$$

But $\mu\left(F^{1 / n}\right) \rightarrow \mu(F)$, so that $\limsup _{i} \mu_{i}(F) \leq \mu(F)+a$, for all $F=\bar{F}$.

From (4.4) this is equivalent to $\mu_{i} \stackrel{a}{\rightarrow} \mu$.

(ii) Let now $\mu_{i} \stackrel{a}{\rightarrow} \mu$ and let $\varepsilon>0$. For every $r>0$ and $t \in T$, let $S_{r}(t)=\{s \in T$ : $d(s, t)<r\}$. Then $\overline{S_{r}(t)} \backslash S_{r}^{\circ}(t) \subseteq\{s \in T: d(s, t)=r\}=C_{r}$. But $C_{r_{1}} \cap C_{r_{2}}=\varnothing$, for all $r_{1} \neq r_{2}$ and $\mu\left(\cup_{r>0} C_{r}\right) \leq 1$. It follows that there exists a countable set $N \subseteq(0,+\infty)$ such that $\mu\left(C_{r}\right)=0$, for all $r \in(0,+\infty) \backslash N$. Therefore, $T$ being separable, there exists a countable family $\left\{S_{r_{n}}\left(t_{n}\right): n \in \mathbb{N}\right\}$ such that (1) $T=\cup_{1}^{\infty} S_{r_{n}}\left(t_{n}\right), \mu\left(\overline{S_{r_{n}}\left(t_{n}\right)} \backslash S_{r_{n}}^{\circ}\left(t_{n}\right)\right)=$ 0 and $r_{n}<\varepsilon / 6$, for all $n \in \mathbb{N}$.

We denote for all $n \in \mathbb{N}, S_{n}=S_{r_{n}}\left(t_{n}\right)$. Let $K \subseteq T$ be a compact set such that $\mu(T \backslash$ $K)<\varepsilon / 3$ and let $p \in \mathbb{N}$ such that $K \subseteq \cup_{n=1}^{p} S_{n}=A_{0}$; then (2) $\mu\left(T \backslash A_{0}\right)<\varepsilon / 3$.

We denote $\mathscr{A}=\left\{\cup_{i=1}^{q} S_{k_{i}}: q \in \mathbb{N}, k_{1}, \ldots, k_{n} \leq p\right\}$; obviously, $A_{0} \in \mathscr{A}$. For every $A \in \mathscr{A}$, $\mu\left(\bar{A} \backslash A^{\circ}\right)=0$ so that, from (4.6),

$$
\underset{i}{\limsup }\left|\mu_{i}(A)-\mu(A)\right| \leq a .
$$

Therefore there exists $i_{0} \in I$ such that, for every $i \geq i_{0}$ and $A \in \mathscr{A}$, (3) $\left|\mu_{i}(A)-\mu(A)\right|<$ $a+\varepsilon / 3$.

Now, for every $F=\bar{F} \subseteq T$, let

$$
A_{F}=\bigcup\left\{S_{n}: n \leq p, S_{n} \cap F \neq \varnothing\right\} \in \mathscr{A} .
$$

Then (4) $F \subseteq A_{F} \cup\left(T \backslash A_{0}\right), A_{F} \subseteq F^{\varepsilon / 3}$.

Indeed, $F=\left(F \cap A_{0}\right) \cup\left(F \backslash A_{0}\right) \subseteq A_{F} \cup\left(T \backslash A_{0}\right)$. For every $t \in A_{F}$ there exists $S_{n}$ such that $t \in S_{n}$ and $S_{n} \cap F \neq \varnothing$. Then, from (1), $d(t, F) \leq 2 \cdot r_{n}<\varepsilon / 3$, so that $t \in F^{\varepsilon / 3}$. Then, from (2), (3), and (4), we have

$$
\begin{aligned}
\mu_{i}(F) & <\mu_{i}\left(A_{F}\right)+\mu_{i}\left(T \backslash A_{0}\right)<\mu(F)+a+\frac{\varepsilon}{3}+1-\mu_{i}\left(A_{0}\right) \\
& <\mu\left(A_{F}\right)+a+\frac{\varepsilon}{3}+1-\mu\left(A_{0}\right)+a+\frac{\varepsilon}{3}=\mu\left(A_{F}\right)+\mu\left(T \backslash A_{0}\right)+2 \cdot a+\frac{2 \varepsilon}{3} \\
& <\mu\left(F^{\varepsilon / 3}\right)+2 \cdot a+\varepsilon \leq \mu\left(F^{\varepsilon}\right)+2 \cdot a+\varepsilon, \\
\mu(F) & \leq \mu\left(A_{F}\right)+\mu\left(T \backslash A_{0}\right)<\mu_{i}\left(A_{F}\right)+a+\frac{\varepsilon}{3}+\frac{\varepsilon}{3} \\
& <\mu_{i}\left(F^{\varepsilon / 3}\right)+a+\frac{2 \varepsilon}{3}<\mu_{i}\left(F^{\varepsilon}\right)+2 \cdot a+\varepsilon,
\end{aligned}
$$

for every $F=\bar{F} \subseteq T$. Then $L_{2 a}\left(\mu_{i}, \mu\right) \leq \varepsilon$, for every $i \geq i_{0}$. Therefore $L_{2 a}\left(\mu_{i}, \mu\right) \rightarrow 0$.

COROLLARY 4.8. Let $H$ be an a-relatively compact subset in $2 \mathfrak{W}^{1}(T)$; then, for every sequence $\left(\mu_{n}\right)_{n \in \mathbb{N}} \subseteq H$, there exist a subsequence $\left(\mu_{k_{n}}\right)_{n \in \mathbb{N}}$ and $\mu \in \mathfrak{2}^{1}(T)$ such that $\mu_{k_{n}} \stackrel{2 \cdot a}{\longrightarrow} \mu$.

Proof. For every sequence $\left(\mu_{n}\right)_{n \in \mathbb{N}} \subseteq H$, there exist a subnet $\left(\mu_{n_{i}}\right)_{i \in I}$ and $\mu \in$ $2 n^{1}(T)$ such that $\mu_{n_{i}} \stackrel{a}{\rightarrow} \mu$. From (4.11), $L_{2 a}\left(\mu_{n_{i}}, \mu\right) \rightarrow 0$. So, for every $p \in \mathbb{N}$, there exists $i_{p} \in I$ such that $n_{i_{p}} \geq p$ and $L_{2 a}\left(\mu_{n_{i_{p}}}, \mu\right)<1 / p$. 
Therefore, we can choose a subsequence $\left(\mu_{n}^{\prime}\right)_{n \in \mathbb{N}}$ of $\left(\mu_{n}\right)_{n \in \mathbb{N}}$ such that $L_{2 a}\left(\mu_{n}^{\prime}, \mu\right) \rightarrow$ 0 . From (4.10) it follows that $\mu_{n}^{\prime} \stackrel{2 \cdot a}{\longrightarrow} \mu$.

Now we are able to give the sequential version of Theorem 3.8.

THEOREM 4.9. Let $\left(\mu_{n}\right)_{n \in \mathbb{N}} \subseteq \mathfrak{V}^{1}(T)$ and $t=t\left(\left\{\mu_{n}: n \in \mathbb{N}\right\}\right)$ be the degree of tightness of $\left(\mu_{n}\right)_{n \in \mathbb{N}}$. Then there exist a subsequence $\left(\mu_{k_{n}}\right)_{n \in \mathbb{N}}$ and $\mu \in 2^{1}(T)$ such that

$$
\mu_{k_{n}} \stackrel{2 \cdot t}{\longrightarrow} \mu
$$

\section{REFERENCES}

[1] P. Billingsley, Convergence of Probability Measures, John Wiley \& Sons, New York, 1968. MR 38 \# 1718. Zbl 172.21201.

[2] N. Bourbaki, Éléments de Mathématique. Fasc. XXXV. Livre VI: Intégration. Chapitre IX: Intégration sur les Espaces Topologiques Séparés, Actualités Scientifiques et Industrielles, no. 1343, Hermann, Paris, 1969 (French). MR 43\#2183. Zbl 189.14201.

[3] P. Brock and D. C. Kent, Approach spaces, limit tower spaces, and probabilistic convergence spaces, Appl. Categ. Structures 5 (1997), no. 2, 99-110. MR 98b:54017.

[4] L. C. Florescu, Probabilistic pseudometrics, Mathematica (Cluj) 24(47) (1982), no. 1-2, 2129. MR 85c:54047.

[5] _ _ A p-complete Menger structure for distribution functions space, An. Ştiinț. Univ. Al. I. Cuza Iaşi Secț. I a Mat. 34 (1988), no. 1, 1-6. MR 89m:54044. Zbl 656.60019.

[6] U. Höhle, Probabilistische Metriken auf der Menge der nicht negativen Verteilungs funktionen, Aequationes Math. 18 (1978), no. 3, 345-356 (German). MR 80k:54057.

[7] R. Lowen, Convergence in a localizable fuzzy topology on spaces of probability measures, Convergence Structures 1984 (Bechyně, 1984), Math. Res., 24, Akademie-Verlag, Berlin, 1985, pp. 217-226. MR 87g:54017.

[8] _ Approach spaces: a common supercategory of TOP and MET, Math. Nachr. 141 (1989), 183-226. MR 90i:54025.

[9] V. Radu, On obtaining Lévy's metric by probabilistic metrics, An. Univ. Timişoara Ser. Ştiinț. Mat. 24 (1986), no. 1-2, 60-65. MR 88j:54046.

[10] R. C. Walker, The Stone-Čech Compactification, Springer-Verlag, New York, 1974, Ergebnisse der Mathematik und ihrer Grenzgebiete, Band 83. MR 52 \# 1595.

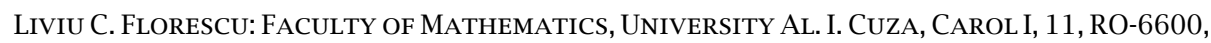
ROMANIA

E-mail address: 1f1o@uaic. ro 


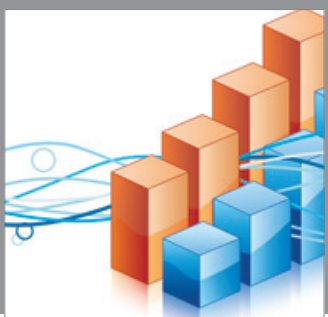

Advances in

Operations Research

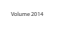

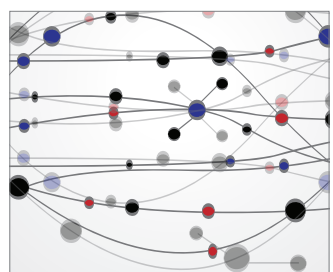

\section{The Scientific} World Journal
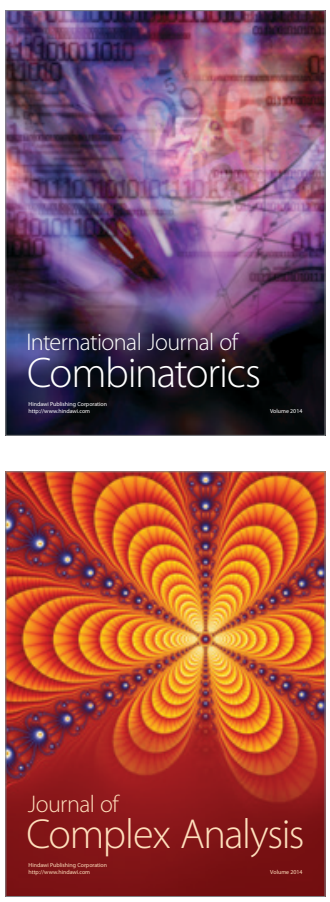

International Journal of

Mathematics and

Mathematical

Sciences
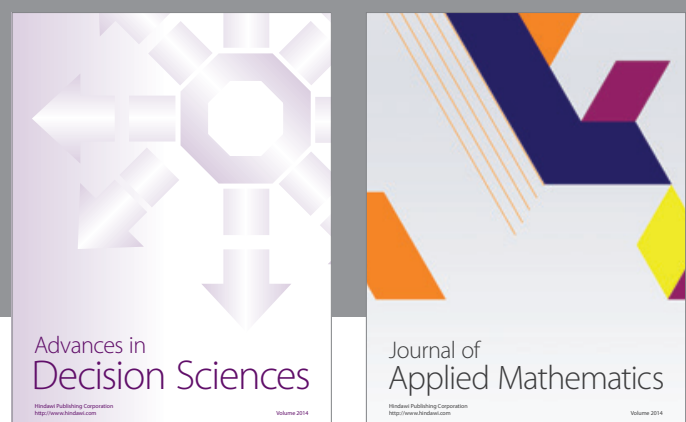

Journal of

Applied Mathematics
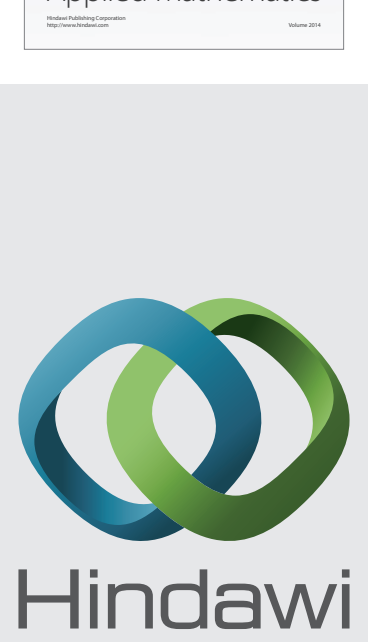

Submit your manuscripts at http://www.hindawi.com
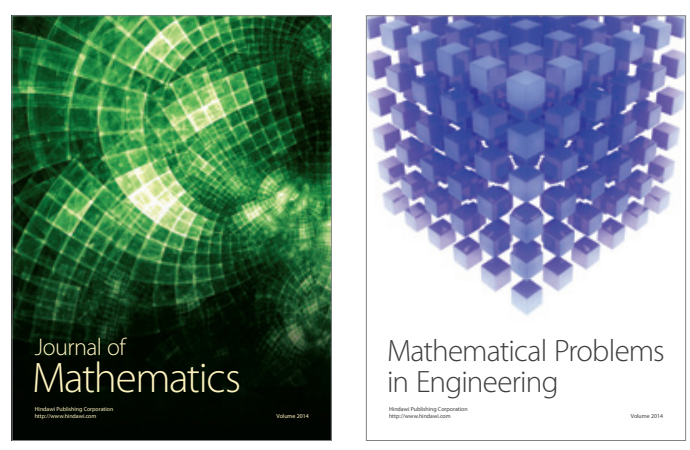

Mathematical Problems in Engineering
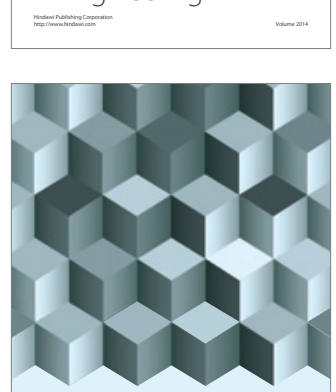

Journal of

Function Spaces
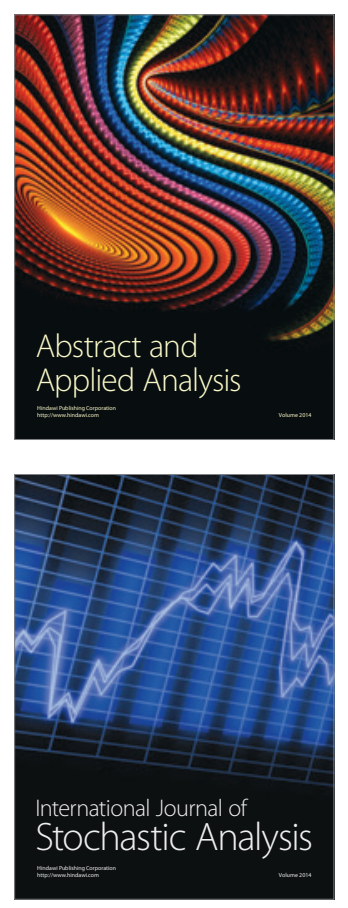

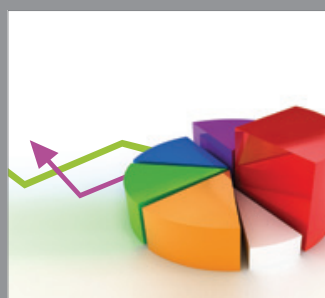

ournal of

Probability and Statistics

Promensencen
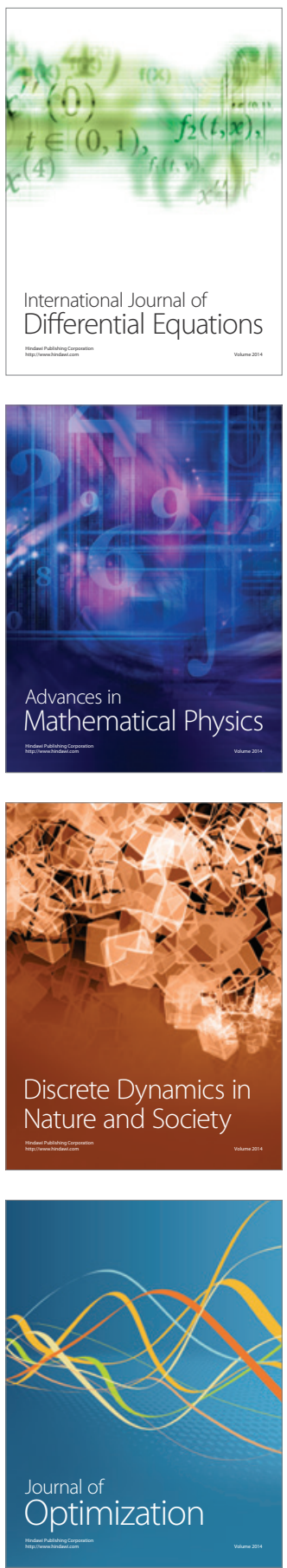